\title{
La reflexión como exigencia en las experiencias de Prácticum de formación inicial
}

\section{de docentes}

\section{Reflection as a requirement in the Practicum experiences of initial teacher training}

\author{
Eduardo José Fuentes Abeledo, Nuria Abal Alonso, Pablo César Muñoz Carril \\ Universidad de Santiago de Compostela
}

\begin{abstract}
Resumen
Esta aportación es producto de un proyecto de investigación, no subvencionado, que estamos desarrollando en los últimos años profundizando en el conocimiento de los procesos y resultados de la formación de maestros y maestras de Educación Infantil en universidades de Galicia. En este trabajo presentamos aspectos de un estudio de caso, centrándonos en los hallazgos en torno a cómo se configura la identidad de una futura docente en relación a la propuesta formativa de la Universidad que apuesta por un modelo de profesor reflexivo e investigador, en un escenario profesional con escasas coincidencias con la visón pedagógica universitaria.

Palabras clave: formación inicial de profesores, prácticum, práctica reflexiva, educación infantil, estudio de caso
\end{abstract}

\begin{abstract}
This contribution is the result of a not-subsidized research project that we are developing in recent years, which endeavors to delve into the knowledge of the processes and outcomes of the training of children's education teachers in Galician Universities. In this paper, we present some aspects of a case study and focus on the findings of how the identity of a future teacher is constructed in relation to the University's formative proposal that opts for a model of a reflective and investigative teacher, in a professional scenario that retains few similarities with the University pedagogical view.

Keywords: initial teacher training, practicum, reflective practice, children's education, case study
\end{abstract}

\section{Contexto, objetivos y metodología}

El proyecto de investigación, no subvencionado, en el que se enmarca esta aportación pretende profundizar en los procesos y resultados de la formación inicial de maestros y maestras de Educación Infantil en el contexto gallego, sirviéndonos de un diseño metodológico que integra tanto el enfoque cuantitativo como el cualitativo $\mathrm{y}$ con un foco prioritario de atención en torno a los aprendizajes que propicia el Prácticum.

En el diseño curricular de la Universidad de Santiago de Compostela de este componente formativo se ha puesto un especial esfuerzo en fundamentar y concretar con coherencia la propuesta desde la Memoria de la
Titulación hasta las actividades específicas a desarrollar en los centros escolares y, en relación con las mismas, aquellas que se realizan en la Universidad bajo la responsabilidad de profesorado universitario. El Prácticum se articula en tres asignaturas (Prácticum I, II y III) desde el segundo al cuarto curso, y ocupa un lugar central para permitir la construcción de las competencias alrededor de la comprensión de la enseñanza y de cómo enseñar, pero también sobre cómo vivir en la escuela y cómo cambiar y mejorar en el desarrollo de la práctica profesional. Los aspectos relacionados con el cambio y la mejora permanentes, tienen mucho que ver con la apuesta, ya expresada en la Memoria de la Titulación, por formar un maestro reflexivo e investigador y que se expresa, con énfasis, en documentos de mayor nivel de concreción. Por ejemplo, entre los aprobados por la Comisión de Prácticum de la Facultad de Ciencias de la Educación de Santiago de Compostela (uno de los dos centros formativos de maestros y maestras de dicha institución académica), se defiende y argumenta en torno a la formación de un profesional "que reflexione sobre su propia práctica, que delibere en el momento de tomar decisiones, que sea capaz de autoevaluarse y de contrastar dicha práctica y las concepciones que la sustentan, enriqueciéndose con las aportaciones de otros docentes y con la investigación sobre muy diversos temas, en un proceso riguroso, sistemático y crítico facilitador además de la construcción de lazos emocionales con diferentes agentes de la comunidad, comprometiéndose en procesos que le permitan "hacer juicios analizando los obstáculos y problemas que pueden estar dificultando el aprendizaje de su alumnado, planificando creativamente ambientes de aprendizaje, experiencias y materiales adecuados conectando escuela y vida haciendo posible el éxito de todos los estudiantes, y practicando una evaluación justa y beneficiosa para el crecimiento de su alumnado y atenta a la mejora de su quehacer profesional y colectivo en el marco de un proyecto educativo de centro" (Fuentes Abeledo, 2011).

En el marco de nuestro proyecto de investigación desarrollamos estudios de caso (Simons, 2011; Mukherji y Algon, 2015), que tienen como protagonistas a alumnos y alumnas de la Universidad de Santiago de 
Compostela y que se centran en la descripción y comprensión del proceso de aprendizaje de la enseñanza de futuros docentes de Educación Infantil, de construcción de su identidad profesional y desarrollo de conocimiento y competencias profesionales. En esta aportación presentamos una primera aproximación a algunos de los aspectos sobresalientes en torno a la construcción de un profesional reflexivo e investigador prestando especial atención a lo acontecido durante el Prácticum II en relación sobre todo con el desarrollo de la competencia "Reflexionar sobre las prácticas de aula propias y ajenas para innovar y mejorar la labor docente relacionando teoría y práctica" (Abal y Fuentes, 2014), aunque haciendo también referencia a aspectos del Prácticum I y en relación con lo producido en el marco de una materia teórico-práctica cursada previamente a las experiencias en centros escolares. Trabajamos sobre el caso "Isabel", nombre que usamos para referirnos a la aprendiz de maestra que ha colaborado en el estudio. Hemos mantenido diversos sistemas de seguridad para mantener el anonimato de los implicados, además de contar con los permisos pertinentes para su realización. Los datos con los que hemos trabajado proceden de múltiples fuentes de las experiencias de Prácticum I (segundo curso) y de Prácticum II (tercer curso) y también en relación con otros momentos y materias del itinerario formativo, en concreto para esta ocasión: cuaderno de reflexión y otros documentos de una materia teórico-práctica ubicada en el mismo cuatrimestre que el Prácticum I (Documento 1) y otros Documentos (D2 y D3) producidos por Isabel (en el contexto del Prácticum I) y de los elaborados en relación a la materia de Prácticum II (D4 y D5). Las experiencias de prácticas en centros fueron realizadas en un aula del segundo ciclo de Educación Infantil.

Para el trabajo con los datos recogidos hemos seguido pautas propias del análisis de contenido: lecturas exploratorias iniciales y posterior codificación y clasificación, para configurar diversas categorías e ir de esta forma penetrando en el significado de las diversas producciones de Isabel. Las categorías y subcategorías reveladas para el conjunto del estudio de caso son numerosas (finalidades de la Educación Infantil, modelo didáctico, profesión docente, clima de aula, currículo, reflexión, investigación, etc.) El proceso seguido nos ha permitido ir desvelando la "perspectiva" de esta futura docente, es decir, sus esquemas de conceptualización, interpretación e intervención en relación con la práctica educativa en Educación Infantil. En esta ocasión nos centramos en aquellos aspectos ligados a los procesos de reflexión e investigación, mostrando evidencias de lo acontecido desde la perspectiva de esta futura docente.

\section{El choque con una realidad diferente a la esperada}

En trabajos anteriores sobre el caso Isabel (Abal, Fuentes y Muñoz, 2015 a y b) ya hemos mostrado cómo en su experiencia de Prácticum I esta futura docente defiende un modelo de trabajo en el aula diferente al que observa. En su opinión, el modelo didáctico vehiculado en la práctica observada no se corresponde con el que define como su "ideal". Isabel defiende un "enfoque de proyectos" que poco tiene que ver con la realidad en la que se sumerge, y que entiende se corresponde mucho más con un modelo "tradicional", "convencional", de "trabajo con fichas" y que describe en variadas ocasiones. Veamos un ejemplo: "Acaban la ficha de preescritura y se disponen en torno a la mesa profesoral para que se les corrija. Se forma una gran algarabía de forma que comienzan las aglomeraciones a la búsqueda del refuerzo que proporcione una satisfacción personal" (D3, 11/8).

Esta realidad choca con la perspectiva ideal que ha ido construyendo en su trayectoria universitaria, ligada a un "enfoque de proyectos" y que, en su opinión, se orienta a convertir el aula en "un espacio de investigación, experimentación, manipulación y observación" (D1, 16/2). Para Isabel el patrón de trabajo habitual que observa tanto en el Prácticum I (con alumnado de 4 años) como en el Prácticum II (con alumnado del último curso de Educación Infantil) se corresponde con un modelo tradicional centrado en la realización de fichas de editoriales con atención muy preferente a contenidos ligados a los procesos específicos de aprendizaje de la lecto-escritura y nociones matemáticas básicas.

\section{Desarrollo de autonomía profesional y orientación hacia la reflexión y la investigación}

Isabel, a pesar de seguir una estrategia de sumisión estratégica (Lacey, 1977) -es decir, acepta y se adapta externamente a lo que dice y hace su tutora, pero mantiene internamente sus propias concepciones que difieren en buena parte de las que sustenta la profesional que la acoge en el aula- logra, a través de un proceso de negociación con esta última, y con apoyo de otra docente que también trabaja unas pocas horas semanales con el mismo grupo-clase de referencia, diseñar y experimentar en el aula un proyecto que, desde su perspectiva, es coherente con su "ideal" de patrón de trabajo en el aula de Educación Infantil y que se identifica, además, con la idea de perfil profesional por la que se decantó ya antes de involucrarse en los escenarios de prácticas. Todo ello, a pesar de que "en un primer momento, mi tutora me forzaba a implementar una propuesta a través de fichas, algo que choca plenamente con mis concepciones como docente" (D4, 36/5).

Las referencias a una docente "innovadora", que "investiga" y "reflexiona" y se convierte en "agente curricular" son abundantes en sus producciones escritas, que estima coherente con su ideal de profesional de la Educación Infantil. Una docente que explora la realidad con la que se encuentra para buscar la mejor forma de potenciar el aprendizaje del alumnado y su más elevada motivación, pero siempre adoptando una posición en la que sus propuestas sean hipótesis a experimentar. Así se manifiesta en diferentes ocasiones, como en un apartado previo a la presentación de su propuesta curricular y en el cual, tras comentar las características de cada niño de su grupo y de las relaciones entre los mismos, ubica su propuesta en la dinámica y trayectoria del grupo y declara que, finalmente, "tuve plena libertad para abordar la educación emocional desde la perspectiva y temática que creyera oportuna. Dado que el alumnado era 
excesivamente heterónomo fui acérrima defensora de la participación guiada orientándola cara a la resolución de conflictos, al conocimiento y las relaciones interpersonales desde una perspectiva empática y transferencial. A pesar de que la temática fuera implícitamente seleccionada por mí, su abordaje iba a ser de gran facilidad pues los niños no tenían desarrollada la capacidad negociadora y democrática en cuanto a la resolución de conflictos y a la toma de decisiones de modo que, al ser sus acciones y conductas sujeto de críticas para el aprendizaje, el alumnado estaría más motivado y dispuesto cara a las actividades asociándome por ende, a un perfil de profesional investigativo" (D4, 37/4).

\section{La práctica de una futura profesora reflexiva e investigadora durante el Prácticum}

El desarrollo de una de las competencias clave antes citada ("Reflexionar sobre las prácticas de aula propias y ajenas para innovar y mejorar la labor docente relacionando teoría y práctica -Abal y Fuentes, 2014-), acorde con las pretensiones del currículo formativo universitario, se refleja en múltiples ocasiones a lo largo de la experiencia de Prácticum II de Isabel. Veamos algunos ejemplos cuando la propia futura docente actúa con autonomía relativa, colaborando en las tareas de su tutora, o cuando lo hace con un alto grado cuando desarrolla la propuesta curricular por ella misma planificada. En los fragmentos seleccionados como ejemplo se relacionan acontecimientos vividos en los que Isabel participa y que ella misma describe, sirviéndose de diversos conceptos y teorías establecidas desde diferentes enfoques, desarrollando interpretaciones propias de lo que sucede, planteando propuestas de intervención, actuando en la práctica de aula y teorizando sobre lo que ocurre.

\section{EJEMPLO 1}

"La teoría del orden de nacimiento de Adler dice que a medida que se incrementa la prole de una familia, la educación parental se va debilitando en pro de los hermanos, pues a medida que los padres van teniendo más hijos adquieren experiencia en el cuidado y educación de estos (learning by doing) otorgándoles una mejor niñez a los hijos que nacen de últimos. En consecuencia, los benjamines, como en este caso, son más mimosos, algo rebeldes y poco previsibles.

- Y tú, ¿quieres venir al cole o prefieres quedarte en casa?

- Pues a veces quiere venir porque estás tú, y otras no porque no me gustan las fichas.

- Ayyyy, a vosotros os gusta venir al cole?

- Sííííí!

- Mucho o poco?

- Muchoooooooo!

- Entonces, ¿qué hacemos para que a Erea le guste el cole y que deje de llorar?

- Tener todas las clases contigo!

- Sííííí!

- Pero eso no puede ser, pobre profe Rosalía, qué haríais entonces con ella?

Ese inconveniente implica que el niño tiene que cambiar el umbral de tolerancia a la frustración pues el ejercicio de autenticidad de la maestra supuso expresar aquello que siente o piensa presentándose tal y como es ante el alumnado.

-Con que nos mande trabajar menos, ya está..., es que a veces trabajamos mucho y si nos portamos mal... nos castiga a hacer fichas...

-Y si se lo digo yo, para que tengáis menos fichas... Erea, ¿tú dejarías de llorar? Y vosotros, ¿estaríais más contentos?

-Sí, porfa.

La apertura y la disposición entendida como la capacidad de escuchar al alumnado es fundamental en la labor de mediación del maestro en cuanto a conocer, comprender y ayudar a sus alumnos, reconociendo el derecho del niño a expresar sus pensamientos y sentimientos y actuando en consecuencia. Esa labor de escucha y de voluntad de cambio (en la medida de las posibilidades), supone una receptividad docente a las necesidades y demandas del alumnado reconociendo que todas las personas necesitan ser escuchadas y valoradas sin hacer juicios morales o de valor al respecto.

-Entonces lo intentaremos, ¿sí?

-Bieeeeeeen!" (D4, 98/8-9 y 99/1-3).

\section{EJEMPLO 2}

"Aprender a ser implica un continuum entre los planos cognitivo, afectivo y social a fin de fomentar en el alumnado un pensamiento autónomo y crítico así como el juicio propio mediante un clima afable, confiado y democrático evitando el enjuiciamiento del alumno. De acuerdo con la observación del alumno, se puede decir que posee una conducta interruptora proclive a la agresión física y verbal como resultado de una inmadurez social y de unas relaciones interpersonales ineficientes. Concretamente, en Bruno convergen diferentes causas para su agresividad instrumental. Es un niño egocéntrico, pues sólo es capaz de ver el mundo únicamente desde su propia óptica teniendo serias dificultades a la hora de descentrarse y empatizar con sus compañeros. Asimismo, la personalidad y el temperamento del propio alumno, derivado de su baja tolerancia a la frustración que desencadena mayor predisposición a conductas disruptivas, inciden directamente en esta situación complementada por un modelo familiar muy permisivo por lo cual el alumnado no tiene límites ni normas a cumplir.

- ¿Tú crees que a tu mamá, a tu papá o a Ana (hermana) les gusta que no tengas amigos porque les pegas? O que te riña la profe?

-No...

$\mathrm{Su}$ ambiente familiar es fundamentalmente permisivo y en una educación sin normas incidiendo directamente en un comportamiento agresivo derivada de la pérdida acentuada del control en la relación con los hijos y el mantenimiento de normas.

$-i \mathrm{Y}$ a ti?

-No.

El proceso mediador deriva de la negociación a través de una persona que actúe como bálsamo neutralizante de las actitudes derivadas. La vida es un marco de referencia en el que prevalecen las normas básicas aunque no siempre igualmente definidas: Rosalía las concibe supeditadas a la autoridad y poder de la maestra, mientras que yo me posiciono en una perspectiva democrática.

-¿Qué podemos hacer para que Bruno sea nuestro amigo de verdad de la buena?

-Que no nos pegue.

-Ni nos tire del pelo.

-Que nos deje trabajar.

-Que comparta los juguetes porque cuando coge algo no lo deja.

-Uy! ¡Cuántas cosas hay que cambiar! ¿no Bruno? A ver: no pegar, compartir, no molestar a los compañeros... ¿Algo más? - ¡Sí! Que nos deje en paz en los recreos porque cuando no queremos nos tira tierra a la cara.

-Y a vosotros, ¿os gusta que haga eso? 


\begin{tabular}{|l|}
\hline -No, para nada! \\
-Y vosotros creéis que Bruno tiene amigos. \\
-No, ninguno... Bueno, André juega con él, a veces, pero \\
siempre están discutiendo. \\
-Y después de escuchar a tus compañeros, ¿tú crees que \\
tienes amigos, Bruno? \\
-No. \\
-Pero nosotros somos tan buenos, tan buenos, tan buenos... \\
Tus compañeros me dijeron que iban a darte una oportunidad \\
PERO... tienes que escucharlos. \\
La capacidad empática permite que el alumno sea capaz de \\
implicarse activamente en la búsqueda de alternativas desde la \\
experiencia común en condiciones de igualdad y desde la \\
resolución específica de conflictos inherentes a la convivencia \\
pues 'la sociedad es conversación y cuanto más pluralista sea \\
aquella, mejor deberá habituarse a prestar oídos a la diferencia \\
en su interior y entablar diálogo con ésta" \\
-Sí porque si no ya no te damos más oportunidades. \\
-Qué hay que hacer entonces para eso, Bruno. \\
-Pues dejar que jueguen cuando quieran conmigo, porque \\
puede que algún día no tengan ganas y si no les pego o les tiro \\
del pelo, al no hacerles pupa van a estar más contentos" \\
(D4,102/7 y D4 103/1-8).
\end{tabular}

Sirvan estos dos fragmentos, de los muchos que cabe rescatar de su producción escrita, para ilustrar la perspectiva de Isabel y cómo establece puentes entre teoría y práctica, desarrollando además teorizaciones sobre su propia práctica y la práctica que observa, sirviéndose, en múltiples ocasiones, de diferentes teorías y resultados de investigación para argumentar en torno a variadas situaciones que se encuentra en la realidad del aula.

La propia Isabel dedica varias páginas a comentar sobre los procesos de teorización, investigación y reflexión del profesorado a partir de su experiencia en el Prácticum, sirviéndose de numerosos autores que han aportado trabajos interesantes sobre estas cuestiones, terminando por afirmar que "la enseñanza es una actividad reflexiva asociada al currículo, a las estrategias docentes y a la formación práctica del profesorado" (D4, 173/5). Y, además, analiza y valora su propio recorrido insistiendo en su esfuerzo por actuar de forma coherente con sus propias ideas y principios: "Desde la concepción progresista y pluralista de la educación fui una 'práctica reflexiva', es decir, una maestra que reflexionó sobre su práctica de un modo intuitivo, flexible y variable de acuerdo a la naturaleza de los distintos problemas planteados en la práctica, motivo por el cual (...) puedo definirme como una facilitadora de aprendizajes de los alumnos y una indagadora crítica. Este proceso amplió las miras del conocimiento profesional, aunque esto no fuera un fin en sí mismo, concibiéndose como un vehículo de mejora del pensamiento y de la toma de decisiones convirtiéndome en una usuaria de conocimiento a la vez que en una productora del mismo. En consecuencia puedo decir que mi proceso de aprendizaje en lo tocante a la práctica educativa estuvo fundamentalmente marcado por una profesionalidad sustentada en la reflexión" (D4, 175/6).

En otro momento, al analizar lo realizado en el marco de su propuesta curricular experimentada y evaluada (Aprendemos a convivir), alude a dos procesos (citando a Perrenoud -2004- y Schön -1992-) para la práctica reflexiva que, según declara, sustentaron su adecuado desarrollo: la reflexión durante el proceso y la reflexión sobre la acción, esta última según manifiesta "apoyada en referencias, teorías subjetivas o científico académicas (Villar, 1992), de forma que la propia persona pueda entender el curso de los acontecimientos" (D4, 148/5).

\section{Observaciones finales}

En un trabajo reciente, hemos vuelto a insistir en la necesidad de definición en los planes formativos universitarios del modelo de profesor y el modelo de formación que fundamenta las decisiones curriculares (Abal, Fuentes y Muñoz, 2017a), comentando diversas opciones posibles (en nuestro caso argumentamos acerca de seis modelos: práctico artesano, especialista en los contenidos de las disciplinas a transmitir, reflexivo e investigador, persona desde una perspectiva humanista, crítico para la justicia social, técnico-experto). En el mismo trabajo comentamos también la necesidad de definir diferentes dimensiones para caracterizar el modelo de referencia en la formación inicial del profesorado de Educación Infantil:

1. PERSONAL Y PROFESIONALIDAD.

2. CONCEPCIÓN DEL APRENDIZAJE Y DE LA ENSEÑANZA.

3. ROL DEL PROFESOR/A.

4. PRÁCTICA CURRICULAR.

5. RELACIONES CON LAS FAMILIAS Y LA COMUNIDAD.

Consideramos muy importante que estas cuestiones, así como un análisis de las competencias profesionales a desarrollar en relación con las distintas funciones y tareas de desempeño profesional, sean trabajadas con las futuras maestras y futuros maestros a lo largo de todo el programa formativo. También que, en coherencia con un modelo de profesor reflexivo e investigador, se establezca un diseño curricular del Prácticum que opte, al configurar las actividades concretas, por propiciar el desarrollo de espirales reflexivas y de investigación-acción.

El caso Isabel muestra cómo, a pesar de unas condiciones de partida en el centro de prácticas poco propicias para el desarrollo de este modelo de profesor y de la configuración de un proceso formativo acorde con el mismo en el Prácticum, es posible, al proponer actividades adecuadas acompañadas de un asesoramiento coherente con el modelo por parte del profesorado universitario (con actividades en la Universidad antes, durante y después de la estancia en los centros y fuerte ligazón con el Prácticum de cuando menos alguna materia teórico-práctica), además de contar también con el entusiasmo por la docencia y un compromiso fuerte con dicho modelo de los propios futuros docentes, desarrollar en profundidad la competencia "Reflexionar sobre las prácticas de aula propias y ajenas para innovar y mejorar la labor docente relacionando teoría y práctica" (Abal y Fuentes, 2014). Ello implica, a nuestro entender, trabajar con los futuros profesores para que identifiquen $\mathrm{y}$ analicen problemas propios de la docencia tanto antes, como durante el Prácticum y estudiarlos desarrollando competencias y actitudes de investigación, sirviéndose 
de teorías y resultados diversos de investigación para comprender los problemas, elaborar hipótesis explicativas y de acción, diseñar planes de acción para resolverlos y experimentarlos en el Prácticum, reflexionar sobre los diversos aspectos implicados sirviéndose también de las evidencias que se recojan en los diferentes momentos de la investigación-acción, y teorizar sobre la práctica que observan y experimentan. Como certeramente ha expresado Judith Irwin (1987, en Brubacher, Case y Reagan, 2000): "El docente reflexivo/analítico toma decisiones sobre la enseñanza partiendo de una conciencia lúcida y de una consideración cuidadosa de 1) los presupuestos que fundamentan sus decisiones y 2) las consecuencias éticas, educativas y técnicas que implican esas decisiones. El maestro puede decidir antes de actuar, durante la acción o después de haber actuado. Para decidir adecuadamente, el docente reflexivo-analítico debe poseer un amplio conocimiento del contenido que va a enseñar, de las opciones teóricas y pedagógicas, de las características de cada alumno y de las restricciones que son producto de la situación en el aula, en la escuela o en la sociedad donde ejerce de la docencia" (pp. 41-42).

Sí hemos de subrayar, sin embargo, que una propuesta formativa que apuesta por un modelo de profesor reflexivo e investigador, necesita, para su cabal implantación, de los recursos humanos y de todo tipo adecuados pues no procede confiar, casi en exclusiva, en la buena voluntad de los agentes intervinientes. Por ejemplo, se requiere que los profesores universitarios cuenten con una adecuada formación y elevado compromiso profesional y que las instancias universitarias reconozcan la carga de trabajo que estas labores conllevan, aspectos que hemos comentado en extenso en otra publicación (Abal, Fuentes y Muñoz, 2017b).

\section{Referencias}

Abal, N. y Fuentes, E. J. (2014). Cuestionario sobre el desarrollo de competencias docentes den la formación inicial del profesorado de Educación Infantil. Facultad de Ciencias de la Educación de la Universidad de Santiago de Compostela. Documento policopiado.

Abal, N; Fuentes, E. J. y Muñoz, P.C. (2015a). Concepciones y construcción de una perspectiva ideal para el trabajo docente en Educación Infantil. En M. Raposo, P.C. Muñoz, M.A. Zabalza-Cerdeiriña, M.E. Martínez y A. Pérez (Coords.), Documentar y Evaluar la experiencia de los estudiantes en las prácticas. Poio 2015 (pp. 339-351). Santiago de Compostela: Anadavira Editora.

Abal, N; Fuentes, E. J. y Muñoz, P.C. (2015b). De la perspectiva ideal a la realidad del aula en las prácticas de futuros docentes de Educación Infantil. En $M$. Raposo, P.C. Muñoz, M.A. Zabalza-Cerdeiriña, M.E. Martínez y A. Pérez (Coords.), Documentar y Evaluar la experiencia de los estudiantes en las prácticas. Poio 2015 (pp. 353-369). Santiago de Compostela: Anadavira Editora.

Abal, N; Fuentes, E. J. y Muñoz, P.C. (2017a). Modelos de profesor y Prácticum: deseos y realidades. En M.
González, M. Raposo, A. Erkizia, M. Cebrián, A. Pérez, M.A. Barberá, O. Canet y M.A. Zabalza (Coords.), Recursos para un prácticum de calidad. Poio 2017 (pp. 291-303). Santiago de Compostela: Anadavira Editora.

Abal, N; Fuentes, E. J. y Muñoz, P.C. (2017b). Desarrollo de competencias y función del supervisor universitario en los procesos de Prácticum. En M. González, M. Raposo, A. Erkizia, M. Cebrián, A. Pérez, M.A. Barberá, O. Canet y M.A. Zabalza (Coords.), Recursos para un prácticum de calidad. Poio 2017 (pp. 291-303) (pp. 279-290). Santiago de Compostela: Anadavira Editora.

Brubacheer, J.W., Case, Ch. W. y Reagan, T.G. (2000). Cómo ser un docente reflexivo. La construcción de una cultura de indagación en las escuelas. Barcelona: Gedisa.

Fuentes Abeledo, E. J. (2011). Documento Marco para o desenvolvemento do Prácticum das titulacións de Mestre. Santiago de Compostela: Comisión de Prácticum da Facultade de Ciencias da Educación da Universidade de Santiago de Compostela. Documento policopiado.

Lacey, C. (1977). The Socialization of Teachers. Londres: Methuen and Co.

Mukherji, P. y Albon, D. (2015). Research Methods in Early Childhood. An Introductory Guide. Londres: Sage.

Perrenoud, Ph. (2004). Desarrollar la práctica reflexiva. Barcelona: Graó.

Schön, D. (1992). La formación de los profesionales reflexivos. Barcelona: Paidós.

Simons, H. (2011). El estudio de caso: Teoría y práctica. Madrid: Morata.

Villar, L.M. (1992). Los profesores y el cambio educativo. Teorías subjetivas y reflexión sobre la práctica. En J.M. Escudero y J. López Yáñez, (Coord.), Los desafios de las reformas escolares. Cambio educativo y formación para el cambio (pp.97-124). Sevilla: Arquetipo. 